\title{
Structural Study and Antibacterial Activity of Cetylpyridinium Dodecyl Sulfate Ion Pair
}

\author{
Oksana Fizer $^{1(\mathbb{D})}$, Mykhajlo Filep ${ }^{2(\mathbb{D})}$, Valerii Pantyo ${ }^{3(\mathbb{D})}$, Danko Elvira ${ }^{4}$ (D) Maksym Fizer $^{1, *(\mathbb{D})}$ \\ 1 Department of Organic Chemistry, Faculty of Chemistry, Uzhhorod National University, Fedinets', Str. 53/1, 88000, \\ Uzhhorod, Ukraine \\ 2 Department of Inorganic Chemistry, Faculty of Chemistry, Uzhhorod National University, Fedinets', Str. 53/1, 88000, \\ Uzhhorod, Ukraine \\ 3 Department of Microbiology, Virology, and Epidemiology with the course of Infectious Diseases, Faculty of Medicine, \\ Uzhhorod National University, Narodna Sq. 1, 88000, Uzhhorod, Ukraine \\ 4 Department of Dental therapy, Faculty of Dentistry, Uzhhorod National University, University Str. 16a, 88000, Uzhhorod, \\ Ukraine \\ * Correspondence: max.fizer@uzhnu.edu.ua (M.F.);
}

Scopus Author ID 55823743600

Received: 19.06.2021; Revised: 23.07.2021; Accepted: 26.07.2021; Published: 9.08.2021

\begin{abstract}
A bactericidal and structural study on the cetylpyridinium dodecyl sulfate ion pair has been taken. The ion pair was a subject of numerous investigations, and its properties are widely discussed in different works. However, systematic studies on the specific interionic interactions in this ion pair are absent in the literature. To fill the gap in knowledge on the structure of cetylpyridinium dodecyl sulfate, we have synthesized this ion pair and characterized it with XRD, NMR, and DFT techniques. Moreover, antibacterial activity against various bacteria strains was analyzed.
\end{abstract}

Keywords: cetylpyridinium; dodecyl sulfate; NMR; DFT; charge transfer.

(C) 2021 by the authors. This article is an open-access article distributed under the terms and conditions of the Creative Commons Attribution (CC BY) license (https://creativecommons.org/licenses/by/4.0/).

\section{Introduction}

Cetylpyridinium salts (chloride and bromide) are widely used as bactericidal cationic surfactants [1-4]. Also, these salts are widely used as a source of the cetylpyridinium cation to synthesize various cetylpyridinium-containing materials [5-9]. One such material is the cetylpyridinium dodecyl sulfate (CPDS) ionic pair, consisting of the two highly lipophilic ions - cetylpyridinium (or 1-hexadecyl pyridinium) cation and the dodecyl sulfate anion. This compound can be obtained via metathesis reaction between cetylpyridinium salts, like chloride (CPC) or bromide (CPB), and sodium dodecyl sulfate (SDS) [9]. Many works of literature mention mixtures of CPC/CPB with SDS, and obviously, CPDS is present in such mixtures [10-12]. It must be mentioned that CPDS is used in ion-selective electrodes as an ionophore. For example, Kulapin and Arinushkina have developed solid-contact potentiometric sensors based on the CPDS associate [13], which were used for the potentiometric determination of cationic and anionic surfactants in hair rinses and shampoos [14]. The authors have used polyvinyl chloride membranes plasticized with dibutyl phthalate, and the CPDS ion pair was used as an ionophore. Moreover, an ion-selective "polyvinyl chloride"-based membrane electrode with CPDS ion pair as ionophore was developed and described by Arvand-Barmchi and co-authors [15]. The electrode with acetophenone plasticizer showed a Nernstian response towards the SDS concentration range from $8.3 \times 10^{-3}$ to $1.0 \times 10^{-6} \mathrm{~mol} / \mathrm{L}$ at $25^{\circ} \mathrm{C}$. 
The systematic investigation of the CPDS ionic pair structure was performed by Maiti and co-authors [9]. The authors have used numerical structure analysis techniques, including NMR; however, despite the in-detail resolved ${ }^{1} \mathrm{H}$ spectrum, the authors have not explained the observed upfield shift of some peaks in the CPDS ion pair ${ }^{1} \mathrm{H}$ spectrum in comparison to the spectra of starting CPC and SDS substances. Interesting in the chemistry of the cetylpyridinium cation $[16,17]$, we have synthesized CPDS and investigated its antibacterial activity in the present study. An intriguing upfield shift in the ${ }^{1} \mathrm{H}$ spectrum of CPDS was found compared with the precursors' CPC and SDS spectra.

\section{Materials and Methods}

All reagents and solvents were purchased from a local supplier, "Sfera Sim," and were used without additional purification. The synthesis of CPDS can be performed according to the known procedure [9]. However, in the present study, the reaction of CPC with SDS was conducted in the water medium, which leads to the formation of a viscous water-CPDS mixture. Further separation of CPDS was done by extraction with chloroform. Obtained chloroform extracts were dried under reduced pressure, which gave white powder of CPDS.

NMR spectra were recorded in deuterated dimethyl sulfoxide (DMSO-d6) solutions on the Varian VXR-300 instrument. DFT computations were performed on the Xeon 20 core workstation with $256 \mathrm{~GB}$ of RAM.

The geometry of the CPDS associate was first optimized with the PM6-D3H4 method $[18,19]$ in the MOPAC2016 program [20]. The water solvation effect was included via the conductor-like screening model COSMO [21]. Further, geometry was reoptimized with the GGA B97-D3 functional [22, 23] with the 6-31G(d,p) basis set [24] on all atoms except oxygen atoms, on which additional diffuse functional has been added. Calculation of electron density for analysis of interionic interactions was performed with the B3LYP functional [25]. Chargetransfer effects were investigated with the range-separated functional CAM-B3LYP [26]. The conductor-like polarizable continuum model (CPCM) [27, 28] has been utilized to account for water solvation effects. All DFT computations were done in the ORCA 4.2 package [29, 30]. The "resolution-of-identity" [31-33] and "chain-of-spheres" [34-36] techniques were turned on to speed up all calculations. A multifunctional wavefunction analyzer Multiwfn 3.8 has been used to analyze electron density files [37]. Visualization of isosurfaces was performed with the VMD [38] package.

X-ray powder diffraction patterns were recorded using the AXRD Benchtop powder diffractometer (Proto Manufacturing Limited) equipped with a hybrid photon counting detector (DECTRIS MYTHEN2 R 1D). Measurements were performed at room temperature in BraggBrentano $\Theta / 2 \Theta$ geometry with $\mathrm{Ni}$-filtered $\mathrm{Cu} \mathrm{K}_{\alpha}$ radiation in 5-70 $2 \Theta$ angle range with a step size of $0.0199^{\circ}$ and counting time 1 s per step. The phase analysis was performed using a PDAnalysis (Proto Manufacturing) and the PowderCell 2.4 software [39], lattice parameters were calculated using the EXPO2014 package [40].

Differential thermal analysis (DTA) was carried out according to the standard technique [41] in the static air atmosphere using a combined chromel-alumel thermocouple (type K) as a temperature sensor and pre-calcined $\mathrm{Al}_{2} \mathrm{O}_{3}$ as reference material. Measurements were performed using an open quartz sample vessel in the range of temperature from room to 500 ${ }^{\circ} \mathrm{C}$ and scan rate of heating at $12^{\circ} \mathrm{C} / \mathrm{min}$. Determination of the temperature of the effects on DTA curves was performed as proposed in [42]. 


\section{Results and Discussion}

\subsection{Structure of the 1:1 CPDS associate.}

The ion-exchange reaction between SDS and CPC water solutions produces the formation of the viscous CPDS solution (Scheme 1). Further extraction with chloroform and evaporation of the organic solvent leads to the white solid with a composition ratio of cetylpyridinium to dodecyl sulfate ions equal 1 to 1 . The electrostatic attraction is expected to be the principal force that combines dodecyl sulfate and cetylpyridinium ions. However, the direct attraction between formally negatively charged oxygen of the sulfo group and formally positively charged nitrogen of the pyridinium cycle is too simplified. That is why it was decided to verify the cetylpyridinium and dodecyl sulfate interactions by analyzing the ion pair's NMR spectrum and electronic structure.

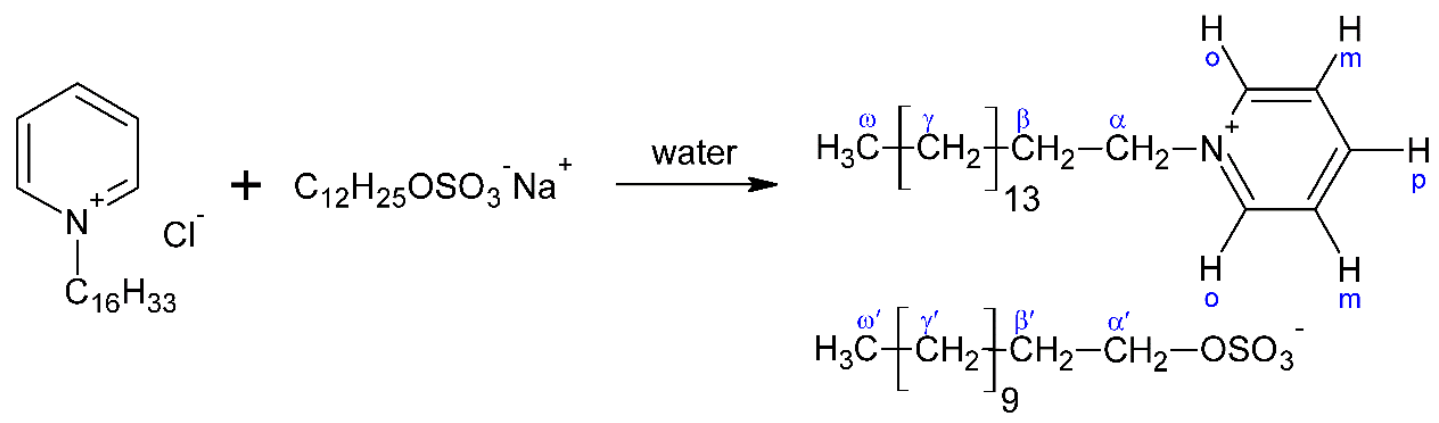

CPDS

Scheme 1. Synthesis of the CPDS ion pair.

To verify the phase homogeneity, the XRD and DTA analysis were taken. Phase analysis was performed by comparing the diffraction patterns of synthesized CPDS with the initial compounds' experimental and simulated diffraction patterns (Fig.1) [6, 9, 43]. The experimental powder pattern does not contain reflexes of initial compounds, which indicates that the synthesized CPDS sample was obtained without impurities.

Indexing of the obtained powder pattern was carried out, and it was established that CPDS crystalize in monoclinic crystal system and the calculated lattice parameters are as follows: $a=10.44 \AA, b=27.47 \AA, c=6.58 \AA, \beta=91.2^{\circ}$, with the cell volume $V=1888.6 \AA^{3}$. The crystallinity of the investigated sample was evaluated by comparing the integrated intensity associated with the amorphous phase to the background, and it is equal to $75.4 \%$.

DTA performed an investigation of the thermal behavior of CPDS in open quartz vessels. A mass measurement accompanied each heating cycle. Maiti and co-authors [9] have conducted the thermal investigation of CPDS in a narrow temperature range of $30-100{ }^{\circ} \mathrm{C}$. To establish the stability range, the heating was carried out to higher temperatures. First, heating was carried out to $500{ }^{\circ} \mathrm{C}$. The DTA heating curve contains five endothermic effects at 69,88 , 96,160 , and $294^{\circ} \mathrm{C}$ (Figure 2). Heating to $500^{\circ} \mathrm{C}$ causes almost complete mass loss.

The experimental heating curve of CPDS was compared with literature data of the thermal analysis of CPC [7] and SDS [44]. The heating curve of CPDS doesn't contain thermal effects characteristic for initial compounds and additionally indicates that synthesized CPDS does not contain impurities. 


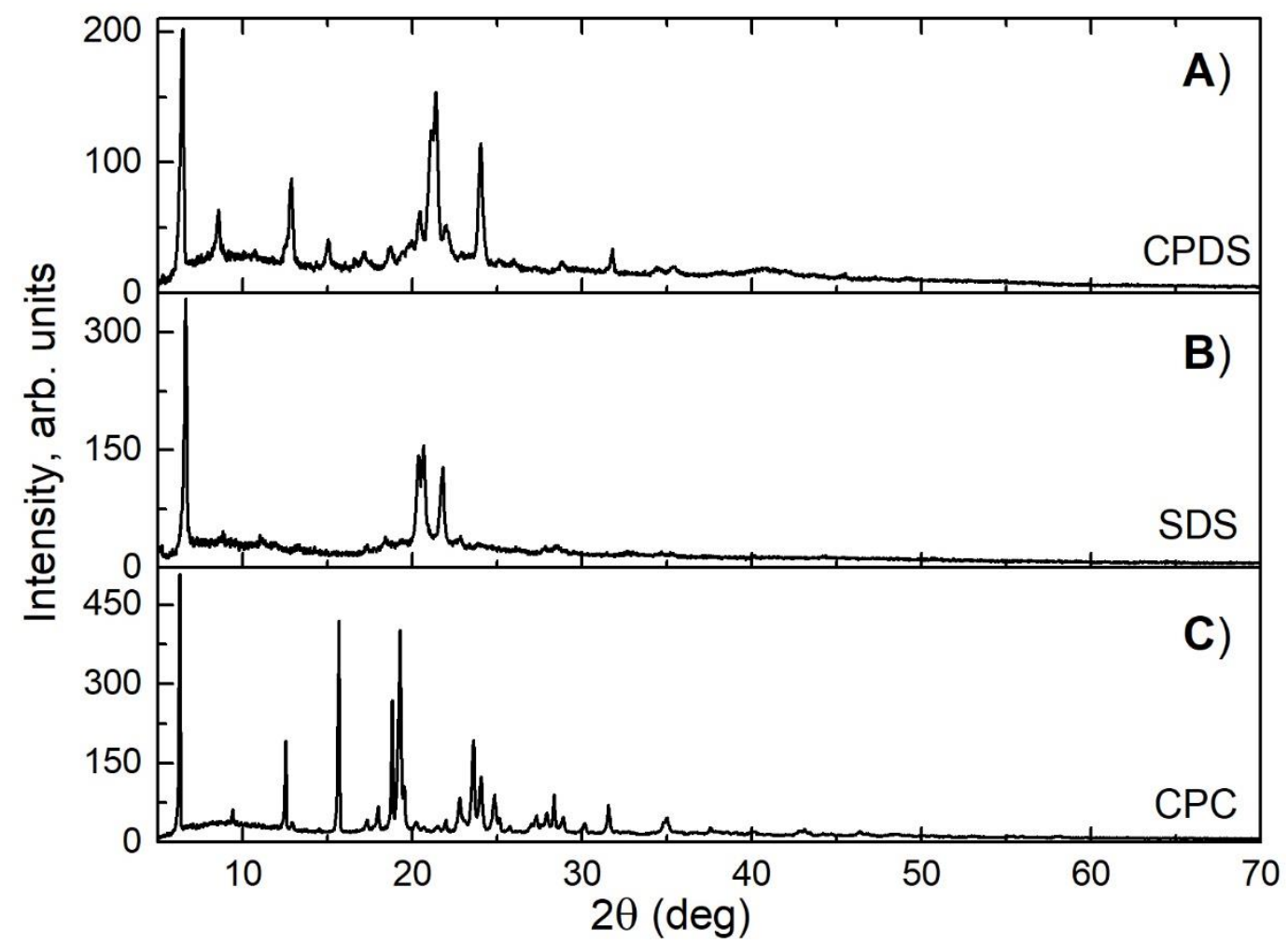

Figure 1. Powder patterns of (A)CPDS; (B) sodium dodecyl sulfate (SDS); (C) cetylpyridinium chloride monohydrate (CPC).

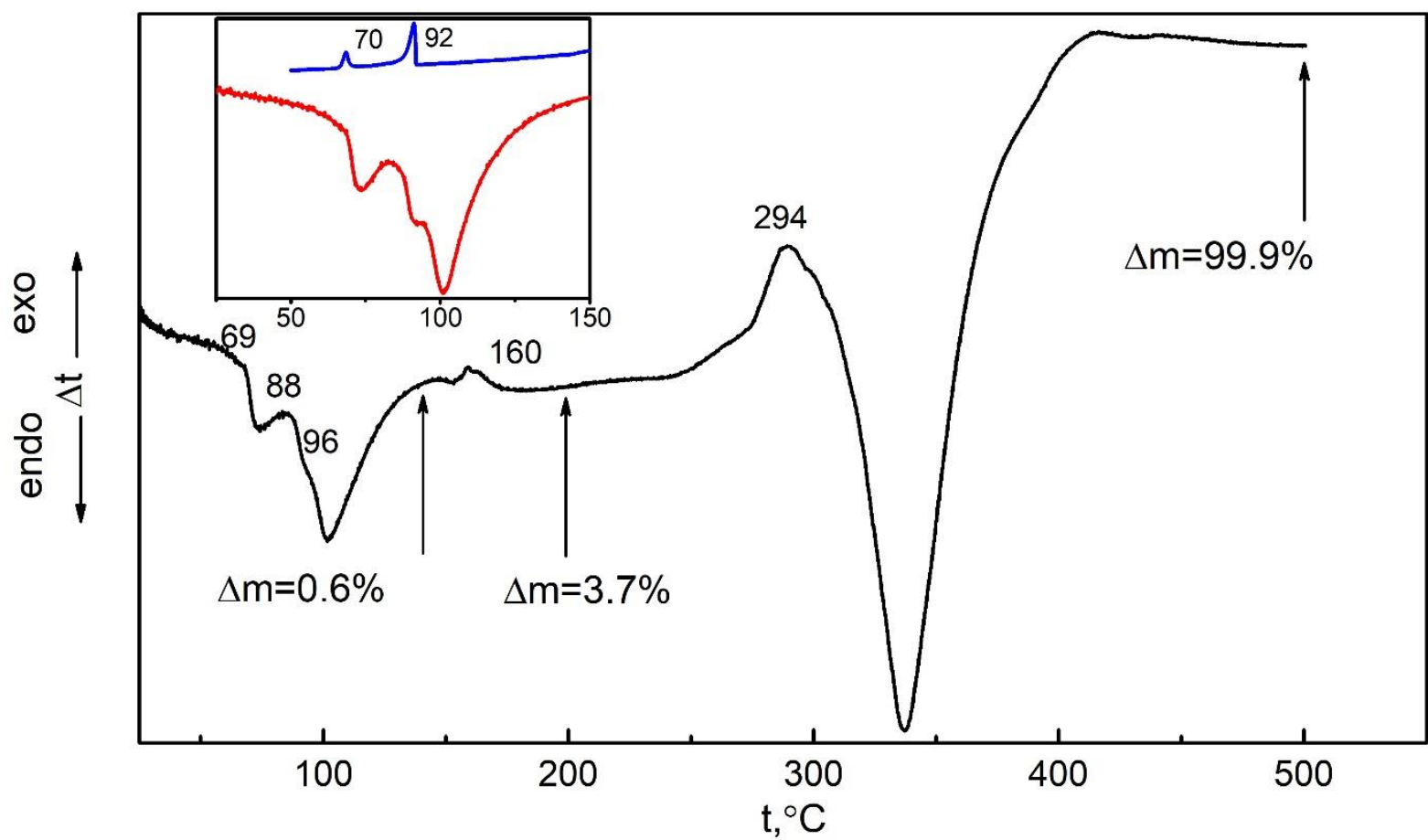

Figure 2. DTA curve of CPDS. The inset window shows heating to $150^{\circ} \mathrm{C}$ (red line) and cooling (blue line) curves.

After the heating to $150{ }^{\circ} \mathrm{C}$, the cooling curve was also recorded. As seen from Fig.2, the cooling curve contains only two exothermic effects at 70 and $92{ }^{\circ} \mathrm{C}$, which agree with the endothermic effects on the heating curve. This indicates the reversibility of the following processes. Obtained data match the results of Maiti and co-authors [9], according to which the first thermal effect corresponds to solid to liquid crystalline phase transition and the second effect is a melting of CPDS. The endothermic effect at $96^{\circ} \mathrm{C}$ doesn't appear on the cooling https://biointerfaceresearch.com/ 
curve. Considering an insignificant mass loss $(\Delta \mathrm{m}=0.6 \%)$, this effect corresponds to the evaporation of adsorbed water. It was heating to $200{ }^{\circ} \mathrm{C}$ causes a mass decrease of $3.7 \%$ and overall appearance (from white powder with a yellow tinge to brown alloy), indicating a partial decomposition of CPDS. Samples obtained after heating to $150{ }^{\circ} \mathrm{C}$ and $200{ }^{\circ} \mathrm{C}$ were also further examined by XRD. The observed powder patterns match with the earlier obtained pattern of as-synthesized CPDS. The strong thermal effect at $294{ }^{\circ} \mathrm{C}$ is caused by vigorous CPDS decomposition and evaporation of formed products $(\Delta \mathrm{m}=99.9 \%)$. It can be summarized that CPDS melts congruently at $88^{\circ} \mathrm{C}$, and it is thermally stable up to $160{ }^{\circ} \mathrm{C}$.

The ${ }^{1} \mathrm{H}$ NMR spectra of the CPDS associate (red spectrum) and CPC (green spectrum) and SDS are presented in Figure 3. Due to the relatively low solubility of CPDS in DMSO, the ${ }^{1} \mathrm{H}$ NMR spectra were recorded for the solution of $8 \mathrm{mg}$ of CPDS in $0.6 \mathrm{~mL}$ of DMSO-d6, which corresponds to $0.014 \mathrm{~mol} / \mathrm{L}$ concentration solution. The DMSO-d6 solutions of CPC and SDS of the same concentration were used for recording the NMR spectra. It is clear that in the upfield region, the signals of aliphatic hydrocarbon groups are located. In the CPDS ion pair case, the triplet at $0.85 \mathrm{ppm}$ with a coupling constant of $6.6 \mathrm{~Hz}$ and relative intensity of about 6 corresponds to two terminal methyl groups of the cetyl and dodecyl chains numerated as $\omega$ and $\omega^{\prime}$ on Scheme 1. The intensive broad peak at $1.24 \mathrm{ppm}$ corresponds to overlapped signals of methylene groups of the cetyl and dodecyl chains - marked as $\gamma$ and $\gamma^{\prime}$ on Scheme 1. A multiplet at $1.65-1.41 \mathrm{ppm}$ is related to the $\beta$ '-methylene group of the dodecyl chain. The multiplet $2.03-1.80 \mathrm{ppm}$ corresponds to the $\beta$-methylene group of the cetylpyridinium cation. The signal of the $\alpha$ '-methylene group of the dodecyl chain appears as a triplet at $3.66 \mathrm{ppm}$ with a coupling constant of $6.7 \mathrm{~Hz}$, and the relative intensity equals 2 . In contrast, the signal of the $\alpha$-methylene group of the cetyl chain appears as a triplet at $4.59 \mathrm{ppm}$ with a coupling constant of $7.4 \mathrm{~Hz}$.

The hydrogens bonded to the pyridinium cycle correspond to the peaks in the spectrum's downfield "aromatic" region. The triplet at $8.16 \mathrm{ppm}$ with the coupling constant of $6.9 \mathrm{~Hz}$ corresponds to the hydrogens in the meta-position of the pyridinium ring. The signal of the para-hydrogen appears as the triplet at $8.60 \mathrm{ppm}$ with the coupling constant of $7.8 \mathrm{~Hz}$. Finally, the signal of the ortho-hydrogens appears as the doublet at $9.08 \mathrm{ppm}$ with the coupling constant of $5.6 \mathrm{~Hz}$.

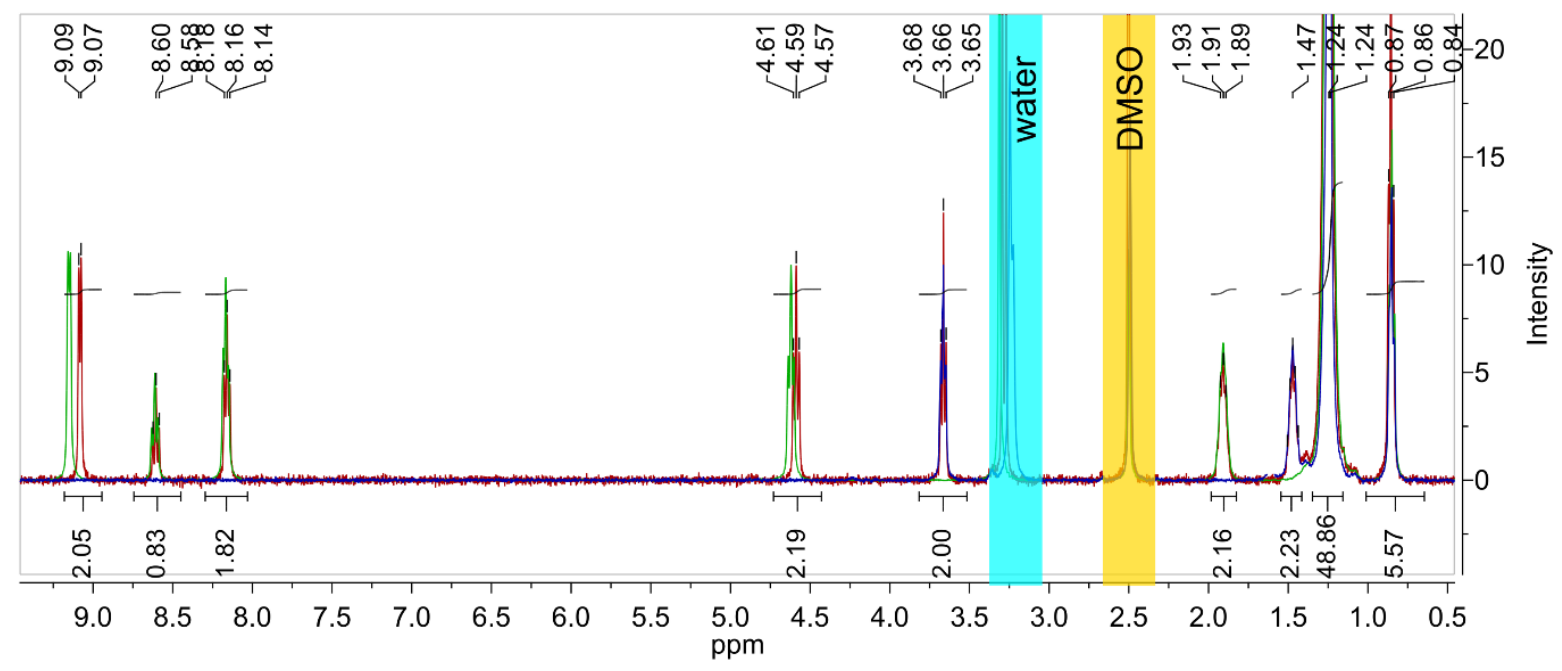

Figure 3. Overlapped ${ }^{1} \mathrm{H}$ NMR spectra of CPDS (red), CPC (green), and SDS (blue). 
It must be noticed that the NMR spectra of the precursor compounds CPC and SDS are almost perfectly matched with the CPDS spectrum. However, in the CPDS spectrum, the signals of the $\alpha-\mathrm{CH} 2$ group and ortho-hydrogen signals are upfield shifted for 0.03 and 0.07 ppm, compared with the CPC spectrum. The upfield shift indicates the strong interionic attraction between these nitrogen-neighboring hydrocarbon groups and the dodecyl sulfate anion. The attraction of electron density from the hydrogen atoms to the oxygens causes deshielding of the protons, which appears as the upfield shift in the NMR spectrum. To verify this explanation, we have performed DFT modeling of the electronic structure of the CPDS ion pair.

\subsection{DFT modeling of the CPDS associate.}

The optimal relative location of ions in the CPDS associate must be chosen to model the CPDS properties accurately. According to the NMR spectra, there is an interaction between the nitrogen-neighboring hydrocarbon groups and oxygen atoms of the dodecyl sulfate anion. That is why the starting geometry was arranged to allow the close location of the $\alpha-\mathrm{CH}_{2}$ group hydrogen and the ortho-hydrogen of the $\mathrm{CP}$ cation and the oxygen atoms of the anion. The alkyl chains remained in an anti-conformation [45], and the two chains were arranged in parallel, which provides maximal van der Waals interactions. After the PM6-D3H4 preoptimization, the CPDS geometry was reoptimized with the B97-D3 functional and the 6$31 \mathrm{G}(\mathrm{d}, \mathrm{p})$ basis set with additional diffuse functions on oxygen atoms; in this study, we will name this basis set as 6-31OG(d,p).

Non-covalent interactions were examined with the reduced density gradient (RDG) isosurface (Figure 4a) [46]. RDG isosurface was calculated based on the B97-D3/6-31OG(d,p) electron density in the Multiwfn program. For example, the red areas on the RDG isosurface in the center of the pyridinium cycle indicate strong electron repulsion. Blue RDG isosurface areas correspond to strong attraction; for example, blue areas between two oxygens of the anion and two hydrogen atoms in the ortho-position of the pyridinium ring and the $\alpha-\mathrm{CH}_{2}$ group. Green areas are related to the van der Waals interactions.
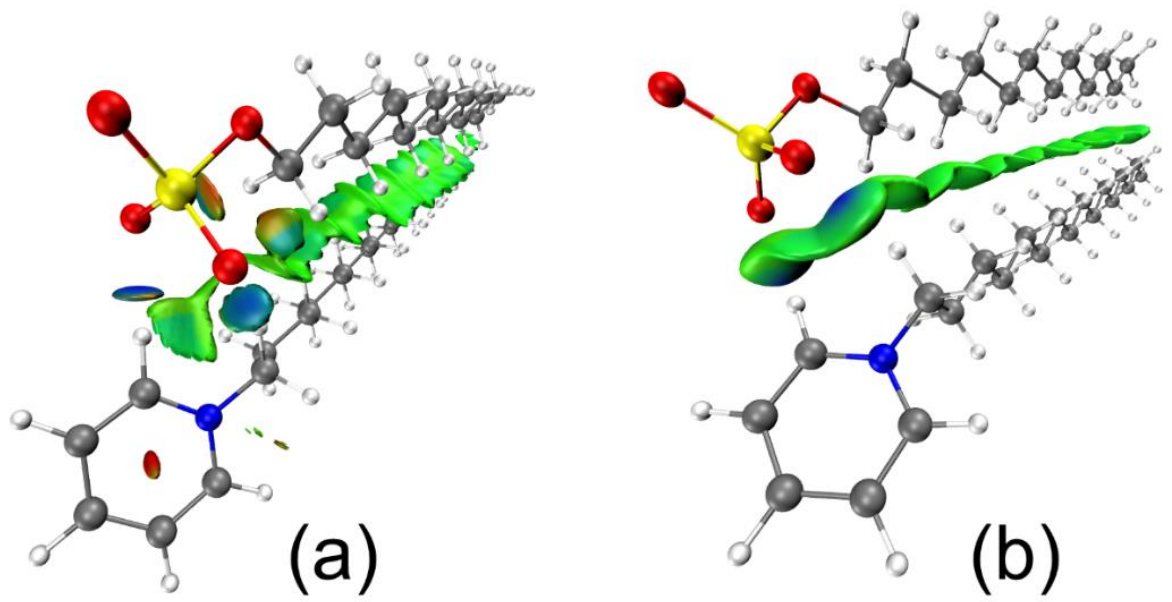

Figure 4. (a) Reduced density gradient (RDG) isosurface; (b) isosurface of independent gradient model (IGM) of interionic interactions.

The independent gradient model (IGM) is another advantageous way for visually studying intermolecular and intramolecular interactions, which was proposed by Lefebvre et al. [47]. In this study, IGM was calculated based on the Hirshfeld partition (IGMH) in Multiwfn according to the scheme proposed by Tian Lu [37]. Interfragment IGM isosurface presented in https://biointerfaceresearch.com/ 
Figure 4b. Two bright blue areas between the $\alpha-\mathrm{CH}_{2}$ group and ortho-hydrogens of the cetyl cation and the anion's oxygens correspond to the strong electrostatic interactions between these atoms. The green area testifies weak dispersion interactions between alkyl chains.

Analysis of inter-fragment interactions could be done in terms of the quantum theory

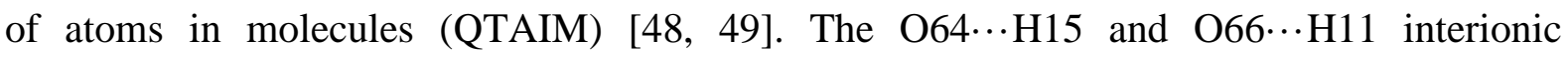
interactions are presented in Figure 5a. The paths are shown as orange lines, critical bond points (BCPs) are shown as green dots, and critical ring points (RCPs) are shown as purple dots. The O64 $\cdots \mathrm{H} 15$ and $\mathrm{O} 66 \cdots \mathrm{H} 11$ BCPs characterized with an electron density of 0.0113 and 0.0169 e/Bohr ${ }^{3}$, respectively; this testifies that the O66 $\cdots \mathrm{H} 11$ interaction is stronger. This finding agrees well with the electrostatic potential maxima located near the ortho-hydrogens in the cetylpyridinium cation [16].
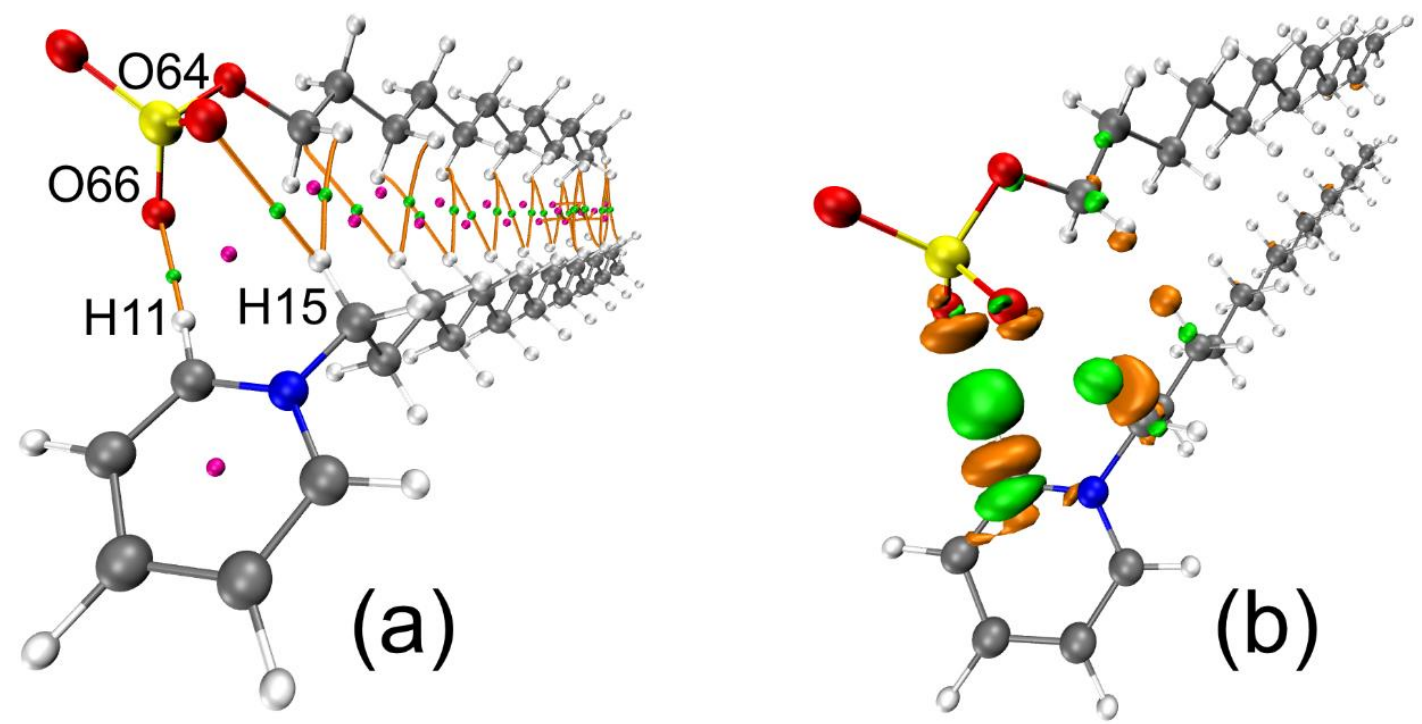

Figure 5. (a) QTAIM analysis of interionic interaction in CPDS; (b) electron density difference during chargetransfer interactions in CPDS.
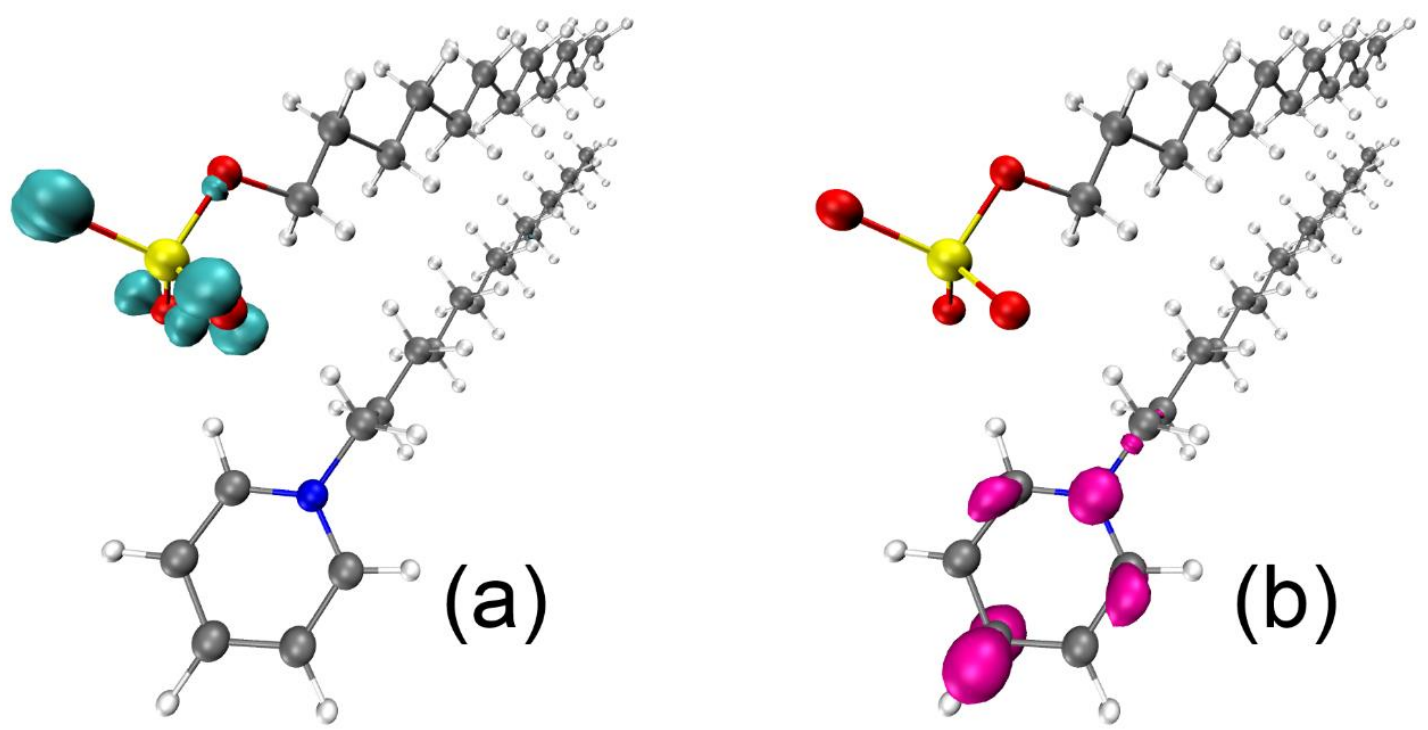

Figure 6. (a) CPDS Fukui function for the electrophilic attack; (b) CPDS Fukui function for the nucleophilic attack.

The O64 $\cdots \mathrm{H} 15$ and $\mathrm{O} 66 \cdots \mathrm{H} 11$ interionic interactions can be characterized with charge transferring (Figure 5b). The green isosurface corresponds to the decrease of electron density 
due to the ions association, whereas the orange isosurface corresponds to the electron density increase. The association is accompanied by the charge-transferring from the ortho-hydrogen of the pyridinium cycle and, to a lesser extent, from the $\alpha-\mathrm{CH}_{2}$ group hydrogen to the $\mathrm{O} 64$ and O66 oxygen atoms of the anion.

Taking into account that the interionic interactions in the CPDS associate are mostly weak van der Waals interactions and strong $\mathrm{O} \cdots \mathrm{H}$ electrostatic interactions, it can be assumed that the nucleophilic-electrophilic character in the associate remains similar to the separate ions. To verify this, we have calculated electrophilic and nucleophilic Fukui functions, which are shown in Figures $6 \mathrm{a}$ and $6 \mathrm{~b}$, respectively. The most suitable sites for the electrophilic attack are the three non-ester oxygen atoms in the dodecyl sulfate anion. In contrast, the preferred locations for the nucleophilic attack are the nitrogen atom and ortho- and para-carbon atoms of the pyridinium ring. These findings support the assumption that the reactivity of the cetylpyridinium and dodecyl sulfate ions in the CPDS associate remains the same.

\subsection{Antibacterial activity of the CPDS associate.}

The antimicrobial activity of the CPDS was tested against the standard strains of E. coli ATCC 29522 and clinical isolates of E. coli, P. vulgaris, K. pneumonia. Quantitative studies to detect the minimum inhibitory concentration (MIC) and the minimum bactericidal concentration (MBC) were performed by serial macro-dilutions in a liquid culture medium. All biological studies were performed twice. A stock solution of CPDS was prepared by dissolving a portion of $0.288 \mathrm{~g}(1 \mathrm{mmol})$ in a mixture of $3 \mathrm{ml}$ of DMSO and $2 \mathrm{ml}$ of ethanol. Thus, the initial concentration of CPDS was $0.2 \mathrm{~mol} / \mathrm{L}$. The cetylpyridinium chloride was used as a reference compound, which was also dissolved in the same mixture of $3 \mathrm{ml}$ of DMSO and 2 $\mathrm{ml}$ of ethanol. DMSO-ethanol (3:2) mixture was used as a control sample.

Table 1. Antimicrobial activity of CPDS, a standard comparison solution of cetylpyridinium chloride, and a control sample of pure DMSO. Sign "+" means the growth of microorganisms in test tubes, and "-" means no

\begin{tabular}{|c|c|c|c|c|c|c|c|}
\hline \multirow[t]{2}{*}{ Compound } & \multirow[t]{2}{*}{ Bacteria } & \multicolumn{6}{|c|}{ Concentration $(\mathrm{mmol} / \mathrm{L})$} \\
\hline & & 50.0 & 25.0 & 12.5 & 6.3 & 3.1 & 1.6 \\
\hline \multirow[t]{4}{*}{ CPDS } & E. coli ATCC 29522 & - & - & - & - & - & + \\
\hline & K. pneumonia* & - & - & - & - & - & + \\
\hline & P. vulgaris* & - & - & - & - & - & + \\
\hline & E. coli* & - & - & - & - & - & + \\
\hline \multirow[t]{4}{*}{$\mathrm{CPC}$} & E. coli ATCC 29522 & - & - & - & - & + & + \\
\hline & K. pneumonia* & - & - & - & - & + & + \\
\hline & P. vulgaris* & - & - & - & - & - & + \\
\hline & E. coli $*$ & - & - & - & - & - & + \\
\hline \multirow[t]{4}{*}{ DMSO } & E. coli ATCC 29522 & - & + & + & + & + & + \\
\hline & K. pneumonia* & - & + & + & + & + & + \\
\hline & P. vulgaris* & + & + & + & + & + & + \\
\hline & E. coli* & + & + & + & + & + & + \\
\hline
\end{tabular}

As shown in Table 1, CPDS showed not lower, but sometimes slightly higher antimicrobial effects than cetylpyridinium chloride. MIC and MBC values for CPCl and CPDS substances were identical for the studied strains of $E$. coli and P. vulgaris and were equal to 3.13 and $6.25 \mathrm{mmol} / \mathrm{L}$, respectively. In the case of E. coli ATCC 29522 and K. pneumonia, the CPDS associate has shown a slightly more pronounced antimicrobial effect. The value of MIC and $\mathrm{MBC}$ to these bacteria were 3.13 and $12.5 \mathrm{mmol} / \mathrm{L}$ and 3.13 and $6.25 \mathrm{mmol} / \mathrm{L}$, respectively, whereas the measured MIC and MBC values for CPC were equal to 6.25 and $25.0 \mathrm{mmol} / \mathrm{L}$ and 
6.25 and $12.5 \mathrm{mmol} / \mathrm{L}$, respectively. We assume that the slightly higher activity of the CPDS ion pair is related to the high lipophilicity of the dodecyl sulfate anion $[50,51]$, which synergistically increases the bioavailability of the cetylpyridinium cation and its better permeability through lipophilic cell membranes. Therefore, we have to suggest that variation of the counter-ion nature in cationic surfactants can increase their antibacterial effect and expand the range of antimicrobial substances with high activity against pathogenic microorganisms.

\section{Conclusions}

The proposed method of synthesis of the cetylpyridinium dodecyl sulfate ion pair gives pure material. According to elemental analysis, XRD and DTA techniques do not contain impurities of the starting reagents. Detailed analysis of ${ }^{1} \mathrm{H}$ NMR spectra of CPDS and starting CPC and SDS compounds shows a specific upfield shift of signals of $\alpha-\mathrm{CH}_{2}$ group hydrogens and the ortho-hydrogens of the cetylpyridinium cation. These shifts indicate an interaction between the nitrogen-neighboring hydrocarbon groups of the cation and the oxygen atoms of the dodecyl sulfate anion. DFT modeling of the geometrical and electronic structure of the CPDS ion pair allowed us to find numerous weak dispersion interactions between the alkyl chains via the RDG, IGM, and QTAIM analysis.

Furthermore, the study of electron density of the associate and separate ions testifies the presence of charge-transfer interactions between the oxygens of the dodecyl sulfate anion and the hydrogens of the cetylpyridinium cation. Electrophilic and nucleophilic Fukui functions support the assumption that the reactivity of the cetylpyridinium and dodecyl sulfate ions in the CPDS associate remains the same, which explains the high antimicrobial effect the CPDS, comparing to the CPC activity. Moreover, a slightly higher antibacterial activity of the CPDS can be explained by the synergistic influence of the dodecyl sulfate anion, which high lipophilicity can increase the bioavailability of the cetylpyridinium cation.

\section{Funding}

This study was partially supported by the Ministry of Education and Science of Ukraine (State Budget Projects 0119U100232 and 0120U100431) and by the Slovak Academic Information Agency (National Scholarship Programme of the Slovak Republic, Grants ID 25718 and 30750).

\section{Acknowledgment}

The authors declare no acknowledgments.

\section{Conflicts of Interest}

The authors declare no conflict of interest.

\section{References}

1. Wu, H.; Yan, Y.; Feng, J.; Zhang, J.; Deng, S.; Cai, X.; Huang, L.; Xie, X.; Shi, Q.; Tan, S. Cetylpyridinium bromide/montmorillonite-graphene oxide composite with good antibacterial activity. Biomed. Mater. 2020, 15, 055002, https://doi.org/10.1088/1748-605X/ab8440. 
2. Mao, X.; Auer, D.L.; Buchalla, W.; Hiller, K.-A.; Maisch, T.; Hellwig, E.; Al-Ahmad, A.; Cieplik, F. Cetylpyridinium chloride: mechanism of action, antimicrobial efficacy in biofilms, and potential risks of resistance. Antimicrob. Agents Chemother. 2020, 64, e00576-20, https://doi.org/10.1128/AAC.00576-20.

3. Alvarez, D.M.; Duarte, L.F.; Corrales, N.; Smith, P.C.; González, P.A. Cetylpyridinium chloride blocks herpes simplex virus replication in gingival fibroblasts. Antiviral Res. 2020, 179, 104818, https://doi.org/10.1016/j.antiviral.2020.104818.

4. Kanno, S.; Hirano, S.; Kato, H.; Fukuta, M.; Mukai, T.; Aoki, Y. Benzalkonium chloride and cetylpyridinium chloride induce apoptosis in human lung epithelial cells and alter surface activity of pulmonary surfactant monolayers. Chem. Biol. Interact. 2020, 317, 108962, https://doi.org/10.1016/j.cbi.2020.108962.

5. Fizer, M.; Fizer, O.; Sidey, V.; Mariychuk, R.; Studenyak, Ya. Experimental and theoretical study on cetylpyridinium dipicrylamide e A promising ion-exchanger for cetylpyridinium selective electrodes. J. Mol. Struct. 2019, 1187, 77-85, https://doi.org/10.1016/j.molstruc.2019.03.067.

6. Fizer, M.; Filep, M.; Fizer, O.; Fricova, O.; Mariychuk, R. Cetylpyridinium picrate: Spectroscopy, conductivity and DFT investigation of the structure of a new ionic liquid. J. Mol. Struct. 2021, 1229, 129803, https://doi.org/10.1016/j.molstruc.2020.129803.

7. Dubovoy, V.; Nawrocki, S.; Verma, G.; Wojtas, L.; Desai, P.; Al-Tameemi, H.; Brinzari, T.V.; Stranick, M.; Chen, D.; Xu, S.; Ma, S.; Boyd, J.M.; Asefa, T.; Pan, L. Synthesis, characterization, and investigation of the antimicrobial activity of cetylpyridinium tetrachlorozincate. ACS Omega 2020, 5, 10359-10365, https://dx.doi.org/10.1021/acsomega.0c00131.

8. Yan, Z.; Cao, X.; Sun, M.; Zhang, L. Physicochemical study on molecular interactions in ternary aqueous solutions of the pharmaceutically active ionic liquid cetylpyridinium salicylate and amino acid/glycylglycine at different temperatures. J. Mol. Liquid. 2021, 326, 115258, https://doi.org/10.1016/j.molliq.2020.115258.

9. Maiti, K.; Bhattacharya, S.C.; Moulik, S.P.; Panda, A.K. Physicochemistry of the binary interacting mixtures of cetylpyridinium chloride (CPC) and sodium dodecylsulfate (SDS) with special reference to the catanionic ion-pair (coacervate) behavior. Colloids Surf. A Physicochem. Eng. Asp. 2010, 355, 88-98, https://doi.org/10.1016/j.colsurfa.2009.11.039.

10. Taş D.S., Çete S. Micellar properties and antimicrobialactivityof a mixed surfactant system constituted by sodium dodecyl sulfate and cetylpyridinium chloride. Indian J. Pharm. Educ. Res. 2017, 51, 580-587, https://doi.org/10.5530/ijper.51.4.87.

11. Singh, O.G.; Ismail, K. Effect of sodium chloride on the aggregation, adsorption and counter-ion binding behavior of mixtures of sodium dioctylsulfosuccinate and sodium dodecylsulfate in water. Colloids Surf. A Physicochem. Eng. Asp. 2012, 414, 209-215, https://doi.org/10.1016/j.colsurfa.2012.08.025.

12. Chanda, S.; Das, D.; Das, J.; Ismail, K. Adsorption characteristics of sodium dodecylsulfate and cetylpyridinium chloride at air/water, air/formamide and air/water-formamide interfaces. Colloids Surf. A Physicochem. Eng. Asp. 2012, 399, 56-61, https://doi.org/10.1016/j.colsurfa.2012.02.024.

13. Kulapin, A.I.; Arinushkina, T.V. Solid-contact potentiometric sensors based on ion pairs of cetylpyridinium with dodecylsulfate and tetraphenylborate for various surfactants. J. Anal. Chem. 2000, 55, 1218-1223, https://doi.org/10.1007/BF02757340.

14. Kulapina, E.G.; Mikhaleva, N.M.; Shmakov, S.L. Separate determination of homologous sodium alkyl sulfates with ion-selective electrodes. J. Anal. Chem. 2004, 59, 487-490, https://doi.org/10.1023/B:JANC.0000026243.39964.44.

15. Arvand-Barmchia, M.; Mousavia, M.F.; Zanjanchib, M.A.; Shamsipurc, M. A new dodecylsulfate-selective supported liquid membrane electrode based on its N-cetylpyridinium ion-pair. Microchem. J. 2003, 74, 149156, https://doi.org/10.1016/S0026-265X(02)00182-0.

16. Fizer, M.; Fizer, O. Theoretical study on charge distribution in cetylpyridinium cationic surfactant. J. Mol. Model. 2021, 27, 203, https://doi.org/10.1007/s00894-021-04820-2.

17. Fizer, O.; Fizer, M.; Sidey, V. Quantum chemical insight on the uranyl benzoates association with cetylpyridinium. J. Radioanal. Nucl. Chem. 2021, https://doi.org/10.1007/s10967-021-07843-4.

18. Stewart, J.J.P. Optimization of parameters for semiempirical methods V: modification of NDDO approximations and application to 70 elements. J. Mol. Modeling 2007, 13, 1173-1213, https://doi.org/10.1007/s00894-007-0233-4.

19. Řezáč, J.; Hobza, P. Advanced corrections of hydrogen bonding and dispersion for semiempirical quantum mechanical methods. J. Chem. Theory Comput. 2012, 8, 141-151, https://doi.org/10.1021/ct200751e. 
20. MOPAC2016, James J. P. Stewart, Stewart Computational Chemistry, Colorado Springs, CO, USA, HTTP://OpenMOPAC.net (2016).

21. Klamt, A.; Schüürmann, G. COSMO: a new approach to dielectric screening in solvents with explicit expressions for the screening energy and its gradient. J. Chem. Soc., Perkin Trans. 2 1993, 1993, 799-805, https://doi.org/10.1039/P29930000799.

22. Grimme, S. Semiempirical GGA-type density functional constructed with a long-range dispersion correction. J. Comput. Chem. 2006, 27, 1787-1799, https://doi.org/10.1002/jcc. 20495.

23. Grimme, S.; Antony, J.; Ehrlich, S.; Krieg, S. A consistent and accurate ab initio parametrization of density functional dispersion correction (dft-d) for the 94 elements H-Pu. J. Chem. Phys. 2010, 132, 154104, https://doi.org/10.1063/1.3382344.

24. Deraet, X.; Woller, T.; Van Lommel, R.; De Proft, F.; Verniest, G.; Alonso, M. A Benchmark of density functional approximations for thermochemistry and kinetics of hydride reductions of cyclohexanones. ChemistryOpen 2019, 8, 788-806, https://doi.org/10.1002/open.201900085.

25. Becke, A.D. Density-functional thermochemistry. III. The role of exact exchange. J. Chem. Phys. 1993, 98, 5648-5652, https://doi.org/10.1063/1.464913.

26. Yanai, T.; Tew, D.P.; Handy, N.C. A new hybrid exchange-correlation functional using the Coulombattenuating method (CAM-B3LYP). Chem. Phys. Lett. 2004, 393, 51-57, https://doi.org/10.1016/j.cplett.2004.06.011.

27. Cossi, M.; Barone, V. Analytical second derivatives of the free energy in solution by polarizable continuum models. J. Chem. Phys. 1998, 109, 6246-6254, https://doi.org/10.1063/1.477265.

28. Takano, Yu; Houk, K.N. Benchmarking the conductor-like polarizable continuum model (CPCM) for aqueous solvation free energies of neutral and ionic organic molecules. J. Chem. Theory Comput. 2005, 1, 70-77, https://doi.org/10.1021/ct049977a.

29. Neese, F. Software update: the ORCA program system, version 4.0. WIREs Comput. Mol. Sci. 2018, 8, e1327, https://doi.org/10.1002/wcms.1327.

30. Neese, F.; Wennmohs, F.; Becker, U.; Riplinger, C. The ORCA quantum chemistry program package. $J$. Chem. Phys. 2020, 152, 224108, https://doi.org/10.1063/5.0004608.

31. Kussmann, J.; Laqua, H.; Ochsenfeld, C. Highly efficient resolution-of-identity density functional theory calculations on central and graphics processing units. J. Chem. Theory Comput.. 2021, 17, 1512-1521, https://doi.org/10.1021/acs.jctc.0c01252.

32. Dou, W.; Chen, M.; Takeshita, T.Y.; Baer, R.; Neuhauser, D.; Rabani, E. Range-separated stochastic resolution of identity: Formulation and application to second-order Green's function theory. J. Chem. Phys. 2020, 153, 074113. https://doi.org/10.1063/5.0015177.

33. Lin, P.; Ren, X.; He, L. Accuracy of localized resolution of the identity in periodic hybrid functional calculations with numerical atomic orbitals. J. Phys. Chem. Lett. 2020, 11, 3082-3088, https://doi.org/10.1021/acs.jpclett.0c00481.

34. Neese, F.; Wennmohs, F.; Hansen, A.; Becker,U. Efficient, approximate and parallel Hartree-Fock and hybrid DFT calculations. A 'chain-of-spheres' algorithm for the Hartree-Fock exchange. Chem. Phys. 2009, 356, 98109, https://doi.org/10.1016/j.chemphys.2008.10.036.

35. Shirazi, R.G.; Pantazis, D.A.; Neese, F. Performance of density functional theory and orbital-optimised second-order perturbation theory methods for geometries and singlet-triplet state splittings of aryl-carbenes. Mol. Phys. 2020, 118, e1764644, https://doi.org/10.1080/00268976.2020.1764644.

36. Maier, T.M.; Ikabata, Y.; Nakai, H. Efficient semi-numerical implementation of relativistic exact exchange within the infinite-order two-component method using a modified chain-of-spheres method. J. Chem. Theory Comput. 2019, 15, 4745-4763, https://doi.org/10.1021/acs.jctc.9b00228.

37. Lu, T.; Chen, F. Multiwfn: a multifunctional wavefunction analyzer. J. Comput. Chem. 2012, 33, 580-592, https://doi.org/10.1002/jcc.22885.46.

38. Humphrey, W.; Dalke, A.; Schulten, K. VMD - visual molecular dynamics. J. Molec. Graphics 1996, 14, 3338, https://doi.org/10.1016/0263-7855(96)00018-5.

39. Kraus, W.; Nolze, G. Powder cell - a program for the representation and manipulation of crystal structures and calculation of the resulting X-ray powder patterns. J. Appl. Crystallogr. 1996, 29, 301-303, https://doi.org/10.1107/S0021889895014920. 
40. Altomare, A.; Cuocci, C.; Giacovazzo, C.; Moliterni, A.; Rizzi, R.; Corriero, N.; Falcicchio, A. EXPO2013: a kit of tools for phasing crystal structures from powder data. J. Appl. Crystallogr. 2013, 46, 1231-1235, https://doi.org/10.1107/S0021889813013113.

41. Hatakeyama, T.; Liu, Z. Handbook of Thermal Analysis, Wiley, 1998.

42. Boettinger, W.J.; Kattner, U.R.; Moon, K.-W.; Perepezko, J.H. DTA and heat-flux DSC measurements of alloy melting and freezing, in: J.-C. Zhao (Eds.), Methods for Phase Diagram Determination, Elsevier Science Ltd, 2007, pp. 151-221, https://doi.org/10.1016/B978-008044629-5/50005-7.

43. Smith, L.A.; Hammond, R.B.; Roberts, K.J.; Machin, D.; McLeod, G. Determination of the crystal structure of anhydrous sodium dodecyl sulphate using a combination of synchrotron radiation powder diffraction and molecular modelling techniques. J. Mol. Struct. 2000, 554, 173-182, https://doi.org/10.1016/S00222860(00)00666-9.

44. Das, A.K.; Saha, S.; Pal, A.; Maji, S.K. Surfactant-modified alumina: An efficient adsorbent for malachite green removal from water environment. J. Environ. Sci. Health A. 2009, 4, 896-905, https://doi.org/10.1080/10934520902958708.

45. Zhang, Y.; Maginn, E.J. Molecular dynamics study of the effect of alkyl chain length on melting points of $\left[\mathrm{C}_{\mathrm{n}} \mathrm{MIM}\right]\left[\mathrm{PF}_{6}\right]$ ionic liquids. Phys. Chem. Chem. Phys. 2014, 16, 13489, https://doi.org/10.1039/c4cp01048e.

46. Johnson, E.R.; Keinan, Sh.; Mori-Sánchez, P.; Contreras-García, J.; Cohen, A.J.; Yang, W. Revealing Noncovalent Interactions. J. Am. Chem. Soc. 2010, 132, 6498-6506, https://doi.org/10.1021/ja100936w.

47. Lefebvre, C.; Rubez, G.; Khartabil, H.; Boisson, J.-C.; Contreras-García, J.; Hénon, E. Accurately extracting the signature of intermolecular interactions present in the NCI plot of the reduced density gradient versus electron density. Phys. Chem. Chem. Phys. 2017, 19, 17928-17936, https://doi.org/10.1039/C7CP02110K.

48. Bader, R.F.W. A quantum theory of molecular structure and its applications. Chem. Rev. 1991, 91, 893-928, https://doi.org/10.1021/cr00005a013.

49. Romani, D.; Brandán, S.A. Investigating the behaviors of corticosterone hormone in different solvents by using DFT calculations and experimental data. Biointerface Res. Appl. Chem. 2020, 10, 4876-4892, https://doi.org/10.33263/BRIAC101.876892.

50. Fizer, O.; Fizer, M.; Sidey, V.; Studenyak, Ya. Predicting the end point potential break values: A case of potentiometric titration of lipophilic anions with cetylpyridinium chloride. Microchem. J. 2021, 160, 105758, https://doi.org/10.1016/j.microc.2020.105758.

51. Fizer, O.; Fizer, M.; Sidey, V.; Studenyak, Ya.; Mariychuk, R. Benchmark of different charges for prediction of the partitioning coefficient through the hydrophilic/lipophilic index. J. Mol. Model. 2018, 24, 141, https://doi.org/10.1007/s00894-018-3692-x. 